\title{
¿Cuántas gaviotas invernan en la costa vasca? Una aproximación basada en censos simultáneos.
}

\section{How many gulls overwinter along the Basque coast? An approach based on simultaneous censuses.}

\author{
Juan Arizaga ${ }^{1 *}$, Asier Aldalur1, Amaia Alzaga ${ }^{2}$, Joseba Amenabar², Óscar Carazo², \\ Sergio Delgado², José F. Esparcia², Aitor Galarza1', Nekane García², Gorka Ocio², \\ Zigor Portu², David Santamaría², Gorka Valdés², Alfredo Valiente ${ }^{2}$, Jon Zubiaur ${ }^{2}$
}

(6)

\section{Resumen}

La costa vasca constituye un área de paso e invernada de gaviotas en el Golfo de Vizcaya y éste a su vez en el Paleártico sudoccidental. Este trabajo se establece con el fin de determinar la importancia relativa de las especies que frecuentan la región en invierno, estimar su abundancia, identificar las zonas más importantes y evaluar hasta qué punto un censo único representa bien la abundancia de gaviotas de un invierno. Estructuralmente, hay dos tipos de estuarios en la costa vasca: aquellos en los que la gaviota reidora Chroicocephalus ridibundus (L. 1766) muestra niveles de abundancia relativa comparables a los de la gaviota patiamarilla Larus michahellis Naumann 1840 y aquellos en los que domina esta última, que por otro lado acumula el $66,5 \%$ de la abundancia de gaviotas en la costa vasca. La estima de la abundancia basada en un modelo log-lineal aportó un valor medio de unas 4500 gaviotas para el invierno de 2019/20 (con una probabilidad al 95\% de que el valor real se sitúe en el rango de 2500 a 7500 gaviotas). En cuanto a las zonas más importantes de la región para la invernada de gaviotas, destacan Donostia, Txingudi, Urdaibai y Ondarroa. Observamos, además, un incremento de la abundancia en días lluviosos respecto a los no lluviosos, si bien este efecto se diluye ante la enorme variabilidad asociada a cada

\footnotetext{
1 Departamento de Ornitología, Sociedad de Ciencias Aranzadi, Zorroagagaina 11, 20014 Donostia.

2 Observador particular. 
zona de muestreo. Esto supone que las cifras son altamente variables, tanto si consideramos el conjunto de especies como cada una de las más frecuentes por separado. Ante esta realidad, se concluye que los resultados de un único censo no representan las abundancias de las gaviotas que invernan en la costa vasca, por lo que se recomienda llevar a cabo varios censos simultáneos con el fin de estimar un valor medio junto a su incertidumbre asociada.

Palabras clave: Golfo de Vizcaya, Cantábrico oriental, censos, ciencia ciudadana, comunidades, invernada, Laridae, Larus.

\begin{abstract}
The Basque coast is a migratory and wintering area for several gull species within the Gulf of Biscay and the Southwestern Palaearctic. This paper aims to assess the relative importance of those species that overwinter along this coast, estimate their abundance, identify the main areas and evaluate to what extent a single census is enough to represent their abundance. Structurally, we found two types of estuaries: those in which the black-headed gull Chroicocephalus ridibundus (L. 1766) co-dominated with the yellow-legged gull Larus michahellis Naumann 1840, and those in which the yellow-legged gull was dominant. The latter species accounted for $66.5 \%$ of the overall gull population size within the Basque coast. The mean gull population size determined by a log-linear model for all species was 4500 individuals for the winter 2019/20 (95\% Cl: 2500-7500 gulls). The main locations within the region were Donostia, Txingudi, Urdaibai and Ondarroa. We observed an increasing number of counts on rainy days as compared to dry days but this effect was very small as compared to the high variance associated with each survey locality. In view of such a high variability, it can be concluded that the results from a single census are not enough to estimate the population size of those gulls overwintering within the region. Several censuses throughout the winter are recommended to obtain a more robust estimate.
\end{abstract}

Key words: The Biscay Bay, Cantabrian region, census, citizen science, communities, wintering, Laridae, Larus.

\title{
Laburpena
}

Euskal kostaldea kaioen eta antxeten paserako eta negua igarotzeko eremua da Bizkaiko Golkoan, eta azken honek funtzio bera betetzen du Mendebaldeko Paleartikoan. Burutu dugun lanaren helburuak izan dira eremu honetan neguan aurki daitezkeen espezieen garrantzi erlatiboa zehaztea, horien ugaritasuna kalkulatzea, zonalde garrantzitsuenak identifikatzea eta kaioen ugaritasuna kalkulatzeko zentso bakar baten egokitasuna ebaluatzea. Egiturari dagokionez, bi motatako estuarioak aurki ditzakegu euskal kostaldean: batetik, antxeta mokogorriaren Chroicocephalus ridibundus (L. 1766) eta kaio hankahoriaren Larus michahellis Naumann 1840 ugaritasun erlatibo antzekoak dituzten estuarioak; eta, bestetik, kaio hankahoriaren, zeinak euskal kostaldeko kaioen \%66,5 osatzen duen, 
ugaritasun erlatibo handiagoa dutenak. Log-lineala ereduan oinarritutako ugaritasun-estimazioak batezbesteko 4500 aleko balioa eman zuen 2019/20 negurako (kaioen kopuru erreala 2500-7500 balioen artean egoteko \% 95eko probabilitatearekin). Negua igarotzeko erabilitako eskualdeei dagokienez, Donostia, Txingudi, Urdaibai eta Ondarroa zonalde garrantzitsu gisa nabarmendu dira. Egindako behaketen arabera, euririk gabeko egunekin alderatuz, egun euritsuetan ugaritasuna hazi egiten da. Alabaina, laginketa-eremuen aldakortasun handia dela-eta efektu horrek indarra galtzen du. Horregatik, jasotako zenbatekoak ere oso aldakorrak izan ohi dira, bai espezie-multzo osoa kontuan izanda, bai maiztasun handieneko espezie bakoitza bere aldetik kontuan hartuta ere. Hori dela eta, zera ondorioztatu da: zentsu bakar bateko emaitzak ez direla esanguratsuak euskal kostaldean negua pasatzen duten kaio eta antxeten ugaritasuna neurtzeko; eta, ondorioz, aldibereko hainbat kontaketa burutzea gomendatzen da, batezbesteko balio bat estimatu ahal izateko, balio horri lotutako ziurgabetasunarekin batera.

Gako hitzak: Bizkaiko Golkoa, Kantauri Ekialdea, zentsoak, herritarren zientzia, komunitateak, negu-pasa, Laridae, Larus.

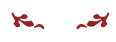

\section{Introducción}

La costa vasca constituye un área de paso e invernada de gaviotas en el Cantábrico y éste a su vez en el Paleártico sudoccidental (Olsen and Larson, 2004). No obstante, esta importancia no ha sido cuantificada hasta la fecha en detalle, más allá del análisis de los datos que se obtienen en los censos de aves acuáticas invernantes llevados a cabo durante una jornada única en enero, además de los dos únicos censos de gaviotas invernantes que se han desarrollado a escala nacional (en la década de 1980 y 2000) y el atlas de aves invernantes de España (Bermejo et al., 1984, Molina, 2009, Fernández et al., 2012, SEO/BirdLife, 2012). Al realizarse en un solo día cabe cuestionarse su representatividad a la hora de estimar la distribución espacial y temporal de gaviotas para el conjunto del invierno en la costa vasca. En este contexto, no se ha comprobado hasta la fecha hasta qué punto dicho censo único de enero representa correctamente la invernada de gaviotas en la región, dada la potencial variabilidad temporal de su abundancia y distribución espacial (J. Arizaga, obs. per.). Respecto a este último caso, no es sólo que las cifras puedan variar al comparar días de anticiclón con temporales (en los que la costa acoge gran número de aves, de otro modo ausentes) (Elkins, 1983). También, aun asumiendo una población más o menos estable puede ser que según factores tales como la disponibilidad de espacios de descanso (mareas, concurrencia de personas y mascotas en playas, etc.) y patrones horarios de alimentación, la presencia de gaviotas en la costa pueda verse alterada. Según la magnitud de la variabilidad debida a estos factores puede ocurrir que el resultado de los 
censos asociado a una jornada sea o no representativo de todo el periodo. Además, el conteo de gaviotas ha sido deliberadamente omitido en algunos puntos de muestreo (Barainka and Arizaga, 2015), lo que puede dar lugar a subestimaciones.

El objetivo de este trabajo es evaluar el papel de la costa vasca como zona de invernada de gaviotas. Concretamente, se pretende (1) determinar la importancia relativa de las especies que frecuentan la región, así como (2) estimar su abundancia, (3) identificar las zonas más importantes y (4) evaluar hasta qué punto un censo único representa bien la abundancia de gaviotas de un invierno. Respecto a este último objetivo, se pretende determinar la variabilidad temporal (esto es, hasta qué punto la población es estable durante el invierno o está sujeta a fluctuaciones muy importantes que, en última instancia, hagan de un censo puntual un hecho irrelevante). En relación a este último objetivo, también se plantea evaluar si el censo de acuáticas de enero es representativo para cuantificar la invernada de gaviotas en la costa vasca.

\section{Material y métodos}

\section{Área de estudio y protocolo de censo}

Este estudio se ha llevado a cabo en las áreas más importantes en las que se conoce presencia habitual de gaviotas en la costa de Euskadi (en adelante, costa vasca) durante el periodo invernal. Se tuvieron en cuenta como puntos de censo aquellas zonas comúnmente usadas por las gaviotas, tanto para alimentarse como para descansar, lo cual incluyó puertos, estuarios y playas, fundamentalmente. En su conjunto, se consideraron 14 puntos de muestreo, desde Txingudi hasta el Abra (Gran Bilbao) (Fig. 1). Según su tamaño, cada zona o "unidad" de muestreo se dividió en sectores, con el fin de organizar mejor el censo (Anexo 1). Por el ejemplo, el estuario del río Urumea se dividió en 11 sectores, situados entre su desembocadura y el barrio de Martutene.

Las 14 unidades de muestreo elegidas incluyeron todas las zonas importantes para gaviotas de la costa de la provincia de Gipuzkoa, pero no así todas las existentes en Bizkaia (Barainka and Arizaga, 2015). En este caso, hubo unidades (e.g. estuarios de Ea, Plentzia o Barbadun) que sí se tienen en cuenta en los censos que se llevan a cabo en enero (Fernández et al., 2012), pero que no pudieron ser muestreados por limitaciones en el número de observadores. En todo caso, según la serie temporal de los censos que se realizan anualmente en enero se trata de zonas donde el número de gaviotas es bajo (Barainka and Arizaga, 2015). En consecuencia, la exclusión de estas zonas no supone, a priori, un sesgo serio en nuestro estudio.

Los censos se realizaron, quincenalmente, durante un periodo de 4 meses entre Nov. de 2019 y Feb. de 2020 (esto es, en total se establecieron, potencialmente, 8 censos en cada unidad de muestreo). Para cada quincena se eligió un sábado durante el cual 


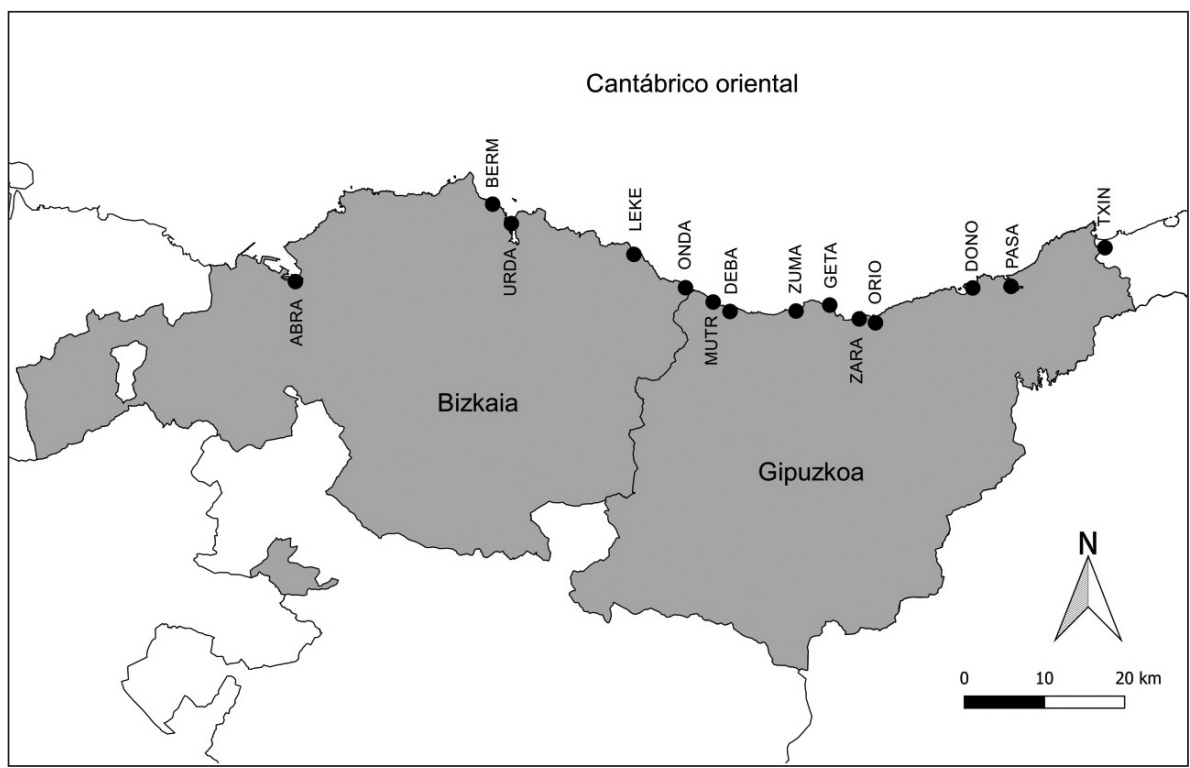

Fig. 1.- Zonas muestreadas para determinar la abundancia de gaviotas invernantes en la costa vasca. Para más detalles ver el Anexo 1.

Fig. 1.- Survey zones along the Basque coast. For further details see the Annex 1.

la bajamar fuera a media mañana. Aproximadamente, los censos se llevaron a cabo durante un periodo de $2 \mathrm{~h}$ en torno a la bajamar, comenzando como pronto a 08:00 y terminando a 12:30. Se tomó como referencia la tabla de mareas del puerto de Pasaia (Gipuzkoa). El motivo de elegir el sábado fue porque el trabajo se realizó por voluntarios que, mayoritariamente, tenían disponibilidad para censar por la mañana en fines de semana. Aunque censar sólo en sábados y durante un horario de mañana puede influir en los resultados (Bermejo et al., 1986), hubo de buscarse un compromiso entre lo mejor desde un punto de vista metodológico y lo posible desde un punto de vista logístico. Los posibles sesgos de este diseño se comentan en la Discusión.

Los censos fueron llevados a cabo por observadores con experiencia en la identificación de gaviotas. Cada observador se responsabilizó de una o más unidades de censo, de tal modo que así se evitaron sesgos en el conteo de cada zona asociados al observador. Para el conteo e identificación de gaviotas se utilizaron binoculares y telescopios terrestres. En cada censo se anotó: (1) el número de ejemplares observados para cada una de las especies; (2) en el caso de gaviota sombría L. fuscus L. 1758 y patiamarilla L. michahellis Naumann 1840, cuando se pudo, se contó el número de aves subadultas (ejemplares que aún no tenían plumaje adulto) y adultas y (3) al meteorología (si llovía o no). 


\section{Análisis estadísticos}

Para determinar el peso de cada zona y quincena en el número de gaviotas se aplicó un modelo lineal generalizado (GLM), en el que el conteo de gaviotas es la variable objeto y la zona, quincena y lluvia son factores. La zona de muestreo podría haberse metido en el modelo como factor aleatorio, ya que (1) hay otras zonas de la costa no muestreadas y (2) cada zona se muestreó en varias ocasiones. No obstante, uno de los objetivos del presente artículo es la identificación de las zonas más importantes para la invernada de gaviotas, motivo por el que se optó por modelos que, explícitamente, consideraran la zona como factor. Debido a la naturaleza de la variable objeto (conteo de individuos), se utilizó una función de enlace log-lineal [esto es, de manera genérica, $\log (\mu)=\alpha+\beta x]$, aplicada en modelos alternativos basados en la distribución de Poisson, quasi-Poisson o binomial negativa. Estas distribuciones, en general se ajustan bien a los datos que resultan del conteo de individuos pero, entre otros aspectos, difieren entre sí en su nivel de 'tolerancia' al tamaño de la varianza (e.g., en la distribución de Poisson la varianza es igual a la media, restricción que no ha de cumplirse en la distribución binomial negativa). Para determinar cuál de estos tres modelos se ajustó mejor a los datos se observó el valor Akaike corregido para muestras pequeñas (AICc) (Akaike, 2011). El ajuste mejora cuanto menor es el valor AIC c de un modelo y diferencias de dos o más unidades Akaike significan diferencias de ajuste entre modelos (Burnham and Anderson, 1998). Se utilizaron los paquetes para R (R Core Team, 2014): "car" "phia" "ImTest" 'MASS'. A partir de los coeficientes del modelo (con su intervalo de confianza al 95\%; IC95\%) se reconstruyó el valor de abundancia por quincena del conjunto de especies y de las especies más frecuentes.

Por otro lado, se llevó a cabo un análisis para determinar la estructura del ensamblado de gaviotas, esto es, el número de especies y la importancia relativa de cada una de las especies. Para ello, primeramente se sumó para cada unidad de muestreo el número de gaviotas censadas, considerando tres grupos: gaviota reidora Chroicocephalus ridibundus (L. 1766), gaviota sombría y patiamarilla y el resto de especies. Partiendo de estos datos, se comprobó la existencia de correlaciones entre la abundancia de estos grupos y el número de especies observadas en cada unidad. Para ello se empleó un test de correlación de Pearson.

Finalmente, con los mismos sumatorios llevados a cabo en el análisis anterior, se procedió a un análisis jerárquico de clusters para determinar la relación entre unidades de muestreo a partir del número de especies detectadas y su abundancia. Este análisis se basó en comparar unidades de muestreo, dos a dos, mediante el índice de Morisita-Horn (Magurran and McGill, 2011). Los clusters se construyeron mediante el método UPGMA a través de la aplicación del programa PAST (Hammer et al., 2001). 


\section{Resultados}

\section{Abundancia}

A lo largo de todo el periodo de estudio se llevaron a cabo un total de 99 censos, de 112 potenciales (14 unidades $\times 8$ jornadas). En 9 unidades de censo se llevaron a cabo el $100 \%$ de los censos que se habían programado (para más detalles ver Tabla 1). El número de ejemplares contados asciende a 26980. Si consideramos sólo los días en que el censo se llevó a cabo en todas las zonas de muestreo, la abundancia media se sitúa en 5060 gaviotas (rango: 3251-6317).

\begin{tabular}{|lrrrrrrrr|}
\hline Zona & Nov1 & Nov2 & Dic1 & Dic2 & Ene1 & Ene2 & Feb1 & Feb2 \\
\hline Txin & 954 & 1280 & 537 & 399 & 665 & 252 & 367 & 355 \\
\hline Pasa & 328 & 182 & 217 & - & 155 & 211 & 127 & 131 \\
\hline Dono & 603 & 503 & 617 & 803 & 779 & 486 & 476 & 378 \\
\hline Oria & 174 & 329 & 320 & - & 355 & 295 & 201 & 159 \\
\hline Zara & 312 & 426 & 127 & 0 & 97 & 20 & 6 & 3 \\
\hline Geta & 174 & 229 & 53 & 40 & 50 & 31 & 45 & 95 \\
\hline Zuma & 189 & 791 & 162 & 389 & 410 & 135 & 200 & 165 \\
\hline Deba & 5 & 397 & 20 & 12 & 35 & 35 & 24 & 36 \\
\hline Mutr & 486 & 32 & 182 & 18 & 11 & 24 & 26 & 24 \\
\hline Onda & 313 & 316 & 398 & 594 & 237 & 157 & 288 & 212 \\
\hline Leke & 476 & 115 & 321 & - & 61 & 109 & 119 & 140 \\
\hline Urda & 1160 & 1144 & - & - & 261 & - & - & - \\
\hline Berm & 297 & 181 & - & - & 70 & - & - & - \\
\hline Abra & 142 & 392 & 798 & 47 & 65 & 182 & 130 & 101 \\
\hline
\end{tabular}

Tabla 1.- Número de gaviotas censadas en cada una de las unidades de muestreo y quincena, entre los meses de noviembre de 2019 y febrero de 2020. Para las abreviaciones ver el Anexo 1.

Table 1.- Number of counts of individual gulls by zone and fortnight for the period November 2019 to February 2020. See Annex 1 for the abbreviations.

El modelo basado en la distribución binomial negativa fue el que mejor se ajustó a los datos (valores AICc: Poisson, 7670; binomial negativa, 1264; no existe AICc para los modelos con distribución quasi-Poisson; sobredispersión: Poisson, 92.23; binomial negativa, 1,18). Según este modelo, la abundancia de gaviotas varía tanto espacial como temporalmente (Tabla 2). No obstante, el modelo en que el factor 'quincena' fue sustituido por el factor 'lluvia' tuvo un ajuste a los datos ligeramente mejor (Tabla 2), por lo que a partir de aquí consideramos este último modelo. Éste mostró valores máximos de abundancia en días de lluvia (Tabla 3). Al reconstruir la abundancia para 


\begin{tabular}{|lcc|}
\hline Parámetros/Estadísticos & Modelo 1 & Modelo 2 \\
\hline Efecto: Zona $(g l=13)$ & $\chi^{2}=130,16 P<0,001$ & $\chi^{2}=127,70 P<0,001$ \\
\hline Efecto: Quincena $(g l=7)$ & $\chi^{2}=58,31 P<0,001$ & $\mathrm{~N} / \mathrm{A}$ \\
\hline Efecto: Lluvia $(g l=1)$ & $\mathrm{N} / \mathrm{A}$ & $\chi^{2}=51,39 P<0,001$ \\
\hline Dispersión & 1,19 & 1,19 \\
\hline AlCc & 1250,5 & 1243,1 \\
\hline
\end{tabular}

Tabla 2.- Resultados de un ANOVA ( $\left(L R \chi^{2}\right.$ y su $P$ asociada), dispersión de Pearson de los datos y valor del estadístico de Akaike (AICC) obtenidos en un modelo lineal generalizado basado en la distribución binomial negativa para determinar el efecto de la zona y la quincena o, alternativamente, de la zona y días de lluvia o no lluvia, en la abundancia de gaviotas en la costa vasca. N/A, No aplica; gl = grados de libertad.

Table 2.- Results from an ANOVA (LR $\chi^{2}$, associated P-value), Person's dispersal and the associated Akaike value (AICC) obtained from a generalized linear model based on a negative binomial distribution used to assess the effect of the zone, fortnight or, alternatively, zone and days with/without rain, on gulls' abundance. N/A, non-applicable; gl, degrees of freedom.

\begin{tabular}{|l|c|c|c|c|c|c|c|c|}
\hline & \multicolumn{2}{|c}{ Total } & \multicolumn{2}{c|}{ Gaviota reidora } & Gaviota patiamarilla & \multicolumn{2}{c|}{ Gaviota sombría } \\
\hline & $\beta \pm \operatorname{SE}(\beta)$ & $P$ & $\beta \pm \operatorname{SE}(\beta)$ & $P$ & $\beta \pm \operatorname{SE}(\beta)$ & $P$ & $\beta \pm \operatorname{SE}(\beta)$ & $P$ \\
\hline Intercepto & $+5,38 \pm 0,07$ & $<0,001$ & $+2,78 \pm 0,14$ & $<0,001$ & $+4,96 \pm 0,08$ & $<0,001$ & $+1,99 \pm 0,14$ & $<0,001$ \\
\hline \multicolumn{7}{|c|}{ Zona1: } \\
\hline Berm & $-0,49 \pm 0,37$ & 0,190 & $-1,89 \pm 0,58$ & 0,001 & $-0,12 \pm 0,38$ & 0,756 & $-1,50 \pm 0,75$ & 0,047 \\
\hline Deba & $-1,26 \pm 0,24$ & $<0,001$ & $-0,09 \pm 0,32$ & 0,798 & $-1,55 \pm 0,25$ & $<0,001$ & $-0,21 \pm 0,45$ & 0,642 \\
\hline Dono & $+1,24 \pm 0,23$ & $<0,001$ & $+2,10 \pm 0,31$ & $<0,001$ & $+1,45 \pm 0,24$ & $<0,001$ & $+1,07 \pm 0,42$ & 0,012 \\
\hline Geta & $-0,89 \pm 0,24$ & $<0,001$ & $-2,74 \pm 0,45$ & $<0,001$ & $-0,57 \pm 0,24$ & 0,018 & $-0,44 \pm 0,46$ & 0,342 \\
\hline Abra & $-0,03 \pm 0,23$ & 0,884 & $+1,34 \pm 0,31$ & $<0,001$ & $-0,28 \pm 0,24$ & 0,234 & $+0,71 \pm 0,43$ & 0,100 \\
\hline Leke & $-0,18 \pm 0,25$ & 0,463 & $-4,73 \pm 0,99$ & $<0,001$ & $+0,08 \pm 0,26$ & 0,802 & $+0,65 \pm 0,45$ & 0,155 \\
\hline Mutr & $-1,08 \pm 0,24$ & $<0,001$ & $+1,14 \pm 0,31$ & $<0,001$ & $-1,34 \pm 0,25$ & $<0,001$ & $-3,07 \pm 0,71$ & $<0,001$ \\
\hline Onda & $+0,59 \pm 0,23$ & 0,011 & $+1,75 \pm 0,31$ & $<0,001$ & $+0,56 \pm 0,24$ & 0,025 & $+1,14 \pm 0,42$ & 0,007 \\
\hline Oria & $+0,37 \pm 0,25$ & 0,112 & $+2,16 \pm 0,33$ & $<0,001$ & $-0,04 \pm 0,26$ & 0,660 & $-1,62 \pm 0,56$ & 0,004 \\
\hline Pasa & $-0,02 \pm 0,25$ & 0,932 & $+1,15 \pm 0,33$ & $<0,001$ & $+0,05 \pm 0,26$ & 0,881 & $-0,12 \pm 0,47$ & 0,808 \\
\hline Txin & $+1,08 \pm 0,23$ & $<0,001$ & $+2,93 \pm 0,31$ & $<0,001$ & $+0,44 \pm 0,24$ & 0,082 & $+1,08 \pm 0,42$ & 0,011 \\
\hline Urda & $+1,02 \pm 0,37$ & 0,006 & $+0,60 \pm 0,48$ & 0,211 & $+1,26 \pm 0,38$ & 0,001 & $+1,50 \pm 0,65$ & 0,022 \\
\hline Zara & $-0,87 \pm 0,24$ & $<0,001$ & $-4,85 \pm 0,98$ & $<0,001$ & $-0,62 \pm 0,24$ & 0,011 & $-0,02 \pm 0,45$ & 0,960 \\
\hline \multicolumn{7}{|c|}{ Lluvia $:$} \\
\hline Lluvioso & $+0,53 \pm 0,07$ & $<0,001$ & $+0,12 \pm 0,10$ & 0,191 & $+0,57 \pm 0,07$ & $<0,001$ & $+1,33 \pm 0,13$ & $<0,001$ \\
\hline
\end{tabular}

$\beta$-parámetros de referencia ( $\beta=0$ ): (1) Zona: Zumaia; (2) Lluvia: No lluvioso. \pm

Tabla 3.- Coeficientes ( $\beta$ parámetros) resultantes de un modelo lineal generalizado desarrollado para determinar la variabilidad espacial y temporal en la abundancia de gaviotas en la costa vasca, en el invierno 2019/20. La función de enlace en el modelo es log-lineal.

Table 3.- Beta-parameter estimates obtained from a generalized linear model used to determine the spatial and temporal variability of the abundance of gulls on the Basque coast, during the winter 2019/20. The link function in the model is log-linear. 


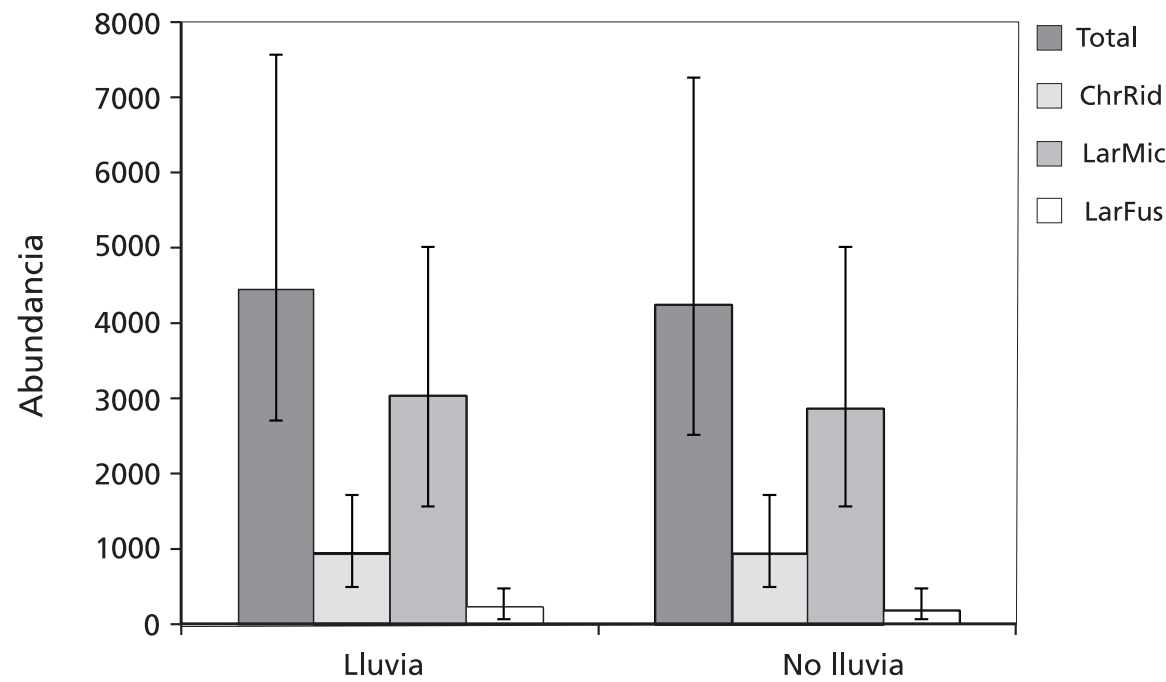

Fig. 2.- Estima ( \pm suma de IC95\%) de la abundancia del conjunto de especies de gaviotas observadas en la costa vasca en el invierno 2019/20, así como de las especies más frecuentes (ChrRid, gaviota reidora; LarMic, gaviota patiamarilla; LarFus, gaviota sombría).

Fig. 2.- Estimate $( \pm 95 \% \mathrm{Cl})$ of all and the most abundant gull species on the Basque coast during the winter 2019/20 (species: ChrRid, black-headed gull; LarMic, yellow-legged gull; LarFus, lesser blackbacked gull).

jornadas de lluvia y no lluvia, no obstante, observamos que la suma de los intervalos de confianza asociados a cada zona fue muy alta por lo que, en consecuencia, el efecto de la meteorología se diluye (Fig. 2). Así, la abundancia estimada de gaviotas (redondeado a \pm 50 ejemplares) se situó en un rango de 4250 ejemplares (IC95\%: 2500-7250) a 4500 ejemplares (IC95\%: 2700-7550) (Fig. 2). Para las especies más abundantes, el modelo repite, grosso modo, los patrones que se observan para la abundancia global, arriba descritos, aunque en el caso de la gaviota reidora no se detectó un efecto de la meteorología en la estimación de su abundancia (Tabla 3). La abundancia global de las especies más abundantes es (para días de lluvia): 200 (IC95\%: 50-500) ejemplares de gaviota sombría, 950 (IC95\%: 500-1700) ejemplares de reidora y 3050 (IC95\%: 1550-5000) ejemplares de patiamarilla.

Tomando Zumaia como referencia (para más detalles ver Tabla 3), hubo 4 unidades de muestreo con una abundancia menor (Deba, Mutriku, Zarautz y Getaria), 4 con una abundancia mayor (Donostia, Txingudi, Urdaibai y Ondarroa), el resto no varió significativamente (Tabla 3). La zona con más abundancia estimada es Donostia (Fig. 3), que junto con las otras con estimas más altas que Zumaia acumulan un $42 \%$ de la abundancia estimada en días lluviosos o un $45 \%$, en días no lluviosos. 


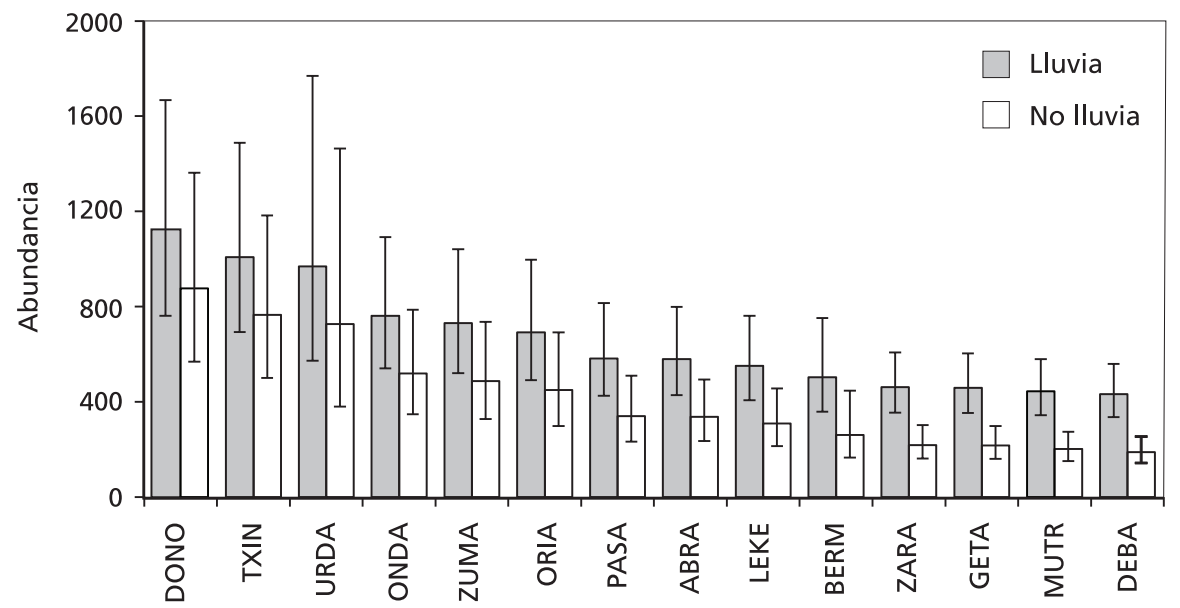

Fig. 3.- Estima ( \pm IC $95 \%$ ) de la abundancia del conjunto de especies de gaviotas observadas en la costa vasca en el invierno 2019/20, en días lluviosos o secos.

Fig. 3.- Estimate $( \pm 95 \% \mathrm{Cl})$ of gull numbers on the Basque coast during the winter 2019/20, between days with/without rain.

En el caso de la gaviota sombría y patiamarilla, la fracción subadulta supone, en promedio, el 32\% (IC95\% = 10\%, $n=26)$ y 43\% (IC95\% = 8\%, $n=29)$ de la población, respectivamente.

\section{Número de especies y estructura del ensamblado}

En conjunto, se detectaron 13 especies de gaviotas (Anexo 2). Globalmente, domina el complejo fuscus/michahellis, el cual aglutina un 72,4\% de la abundancia (19429 aves contadas en la suma de censos). La gaviota reidora suma el 25,6\% (6882 aves contadas) y el resto de especies, el 2,0\% (544 aves). En el complejo fuscus/michahellis, la gaviota patiamarilla alcanza el $91,6 \%$ de la abundancia de las dos especies (o lo que es lo mismo el 66,5\% de la abundancia global). La abundancia relativa de especies varía entre unidades de muestreo (Fig. 4). Así, el complejo fuscus/michahellis domina en 12 unidades; en cinco de ellas es, además, casi el único grupo de gaviotas presente (Zarautz, Getaria, Lekeitio, Urdaibai y Bermeo).

Teniendo en cuenta la presencia y la abundancia de cada una de las especies, la invernada de gaviotas en la costa vasca se estructura en 3 grandes bloques (Fig. 5): (1) las zonas con dominancia de gaviota reidora o codominancia de ésta con el complejo fuscus/michahellis (Txingudi, Oria y Mutriku); (2) las cinco zonas mencionadas anteriormente, en las que más del $95 \%$ de la abundancia se debe al complejo 


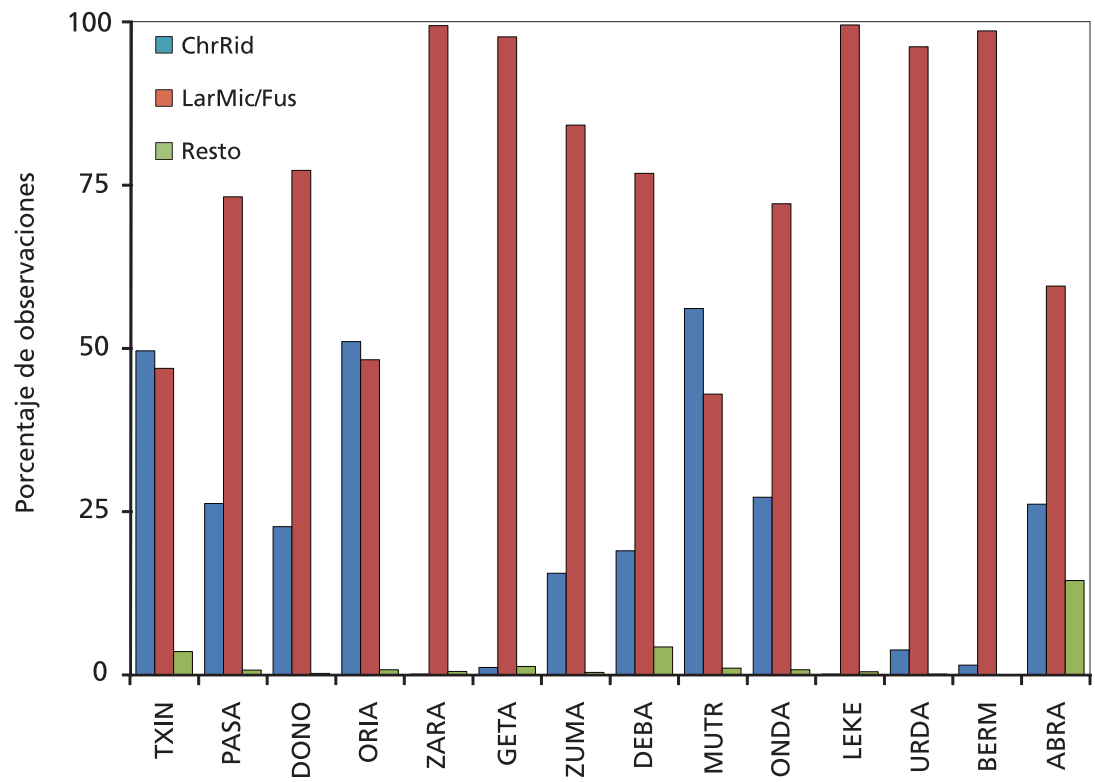

Fig. 4.- Abundancia relativa de gaviota reidora (ChrRid), gaviota sombría-patiamarilla (LarMic/Fus, también llamado complejo fuscus/michahellis en el artículo) y el resto de gaviotas censadas durante el estudio. Las unidades de muestreo están en orden, de este a oeste, según se muestran en la Fig. 1.

Fig. 4.- Relative abundances of the black-headed gull (ChrRid), lesser black-backed/yellow legged gull (LarMic/Fus, also called fuscus/michahellis complex in this work), and the rest of gulls counted over the study period. Sampling localities shown in order (from east to west), as in Fig. 1.

fuscus/michahellis y (3) el resto de zonas, donde domina el complejo fuscus/michahellis, pero en el que también hay gaviota reidora en porcentajes que van del 15,5\% (Zumaia) al 27,2\% (Ondarroa), esto es, en torno al 20\%.

La relevancia numérica de especies al margen de la gaviota reidora, sombría y patiamarilla es bajísima. En 9 unidades de muestreo está por debajo del 1\%, en 5 por debajo del $5 \%$ y sólo en el Abra alcanza el 14,4\%. Esto se debe, parcialmente, a una jornada muy excepcional en la que se contaron 170 gaviotas cabecinegras Larus melanocephalus, que si son eliminadas del análisis disminuyen este porcentaje al 5,7\%.

El número de especies detectadas en cada zona no se correlacionó significativamente ni con la abundancia relativa de gaviota reidora $(r=+0,35, P=0,218)$ ni con la del complejo fuscus/michahellis $(r=-0,45, P=0,104)$, pero sí, en cambio, con la abundancia relativa registrada para el resto de especies $(r=+0,65, P=0,012)$ (Fig. 6). 

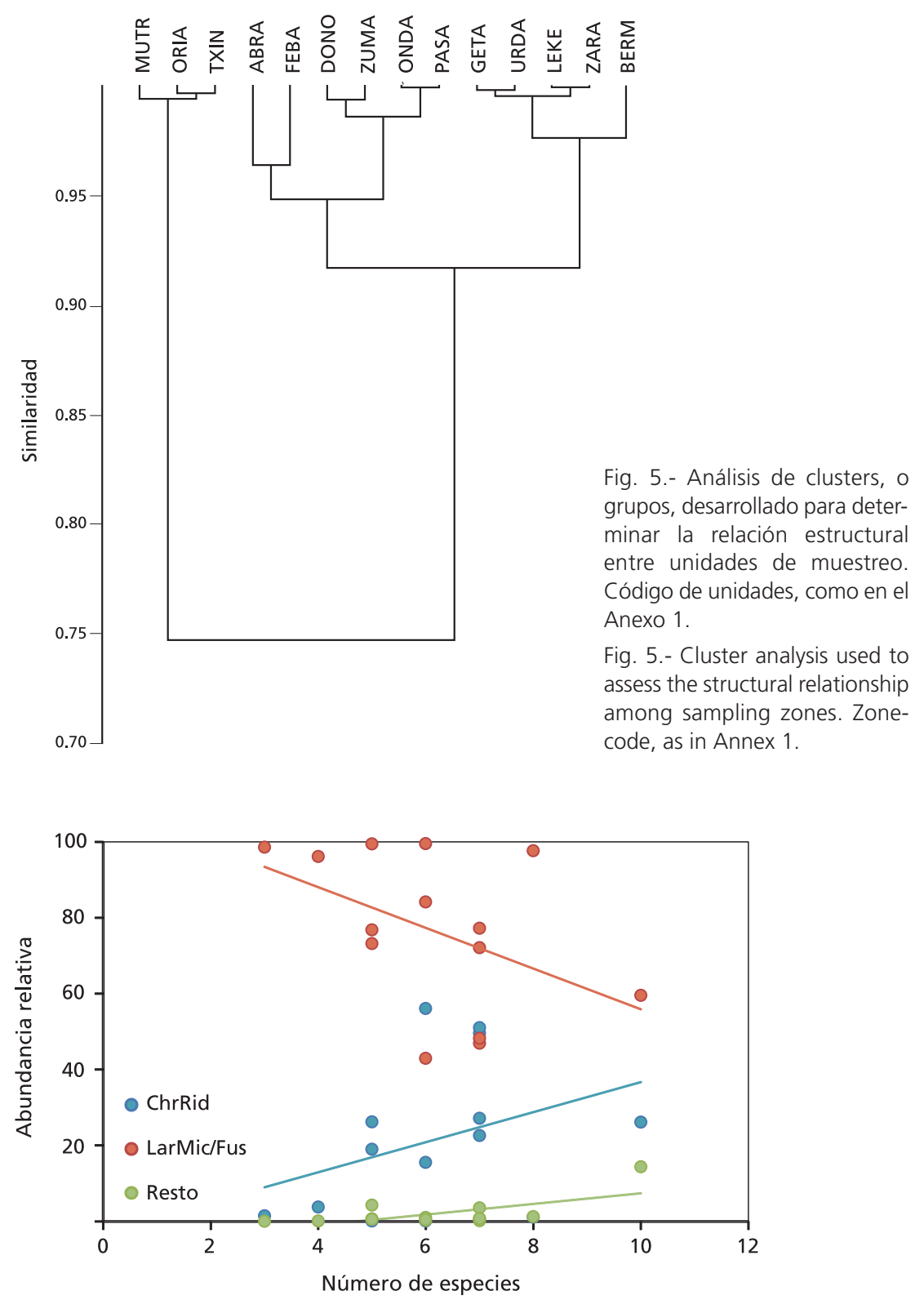

Fig. 6.- Diagrama de correlación de la abundancia relativa de gaviota reidora (ChrRid), gaviota sombría-patiamarilla (LarMic/Fus) y el resto de gaviotas censadas durante el estudio, en relación al número de especies detectadas en cada unidad de muestreo.

Fig. 6.- Linear relationships of the relative abundances of black-headed gulls (ChrRid), lesser blackbacked/yellow-legged gull complex (LarMic/Fus) and the rest of gull species counted, in relation to the number of species detected in each sampling zone. 


\section{Discusión}

Este es el primer estudio en el que se analiza la variabilidad espacial y temporal de la abundancia de gaviotas invernantes en la costa vasca, pues hasta la fecha sólo se disponía de los datos del censo de enero asociado al conteo internacional de aves acuáticas (Fernández et al., 2012), así como los dos censos de gaviotas invernantes llevados a cabo a escala nacional en la década de 1980 y 2000 y el atlas de aves invernantes de 2012 (Bermejo et al., 1984, Molina, 2009, SEO/BirdLife, 2012). En todos estos casos no se abordó el problema de la variabilidad temporal de la abundancia.

La estima de la abundancia basada en un modelo log-lineal aportó un valor medio de unas 4500 gaviotas para el invierno de 2019/20 (con una probabilidad al 95\% de que el valor real se sitúe en el rango de 2500 a 7500 gaviotas). Este valor estimado varía poco en relación a las poco más de 5000 gaviotas contadas los días en que el censo se llevó a cabo en todas las zonas de muestreo (esto es, los días sin unidades de muestreo no censadas) y entra en el rango obtenido para la estima global. Observamos, además, un incremento de la abundancia en días de lluvia respecto a jornadas de tiempo no lluvioso, si bien este efecto se diluye ante la enorme variabilidad asociada a cada zona de muestreo. Esto supone que las cifras son altamente variables, tanto si consideramos el conjunto de especies como las especies más frecuentes por separado. Ante esta realidad, cabe concluir que los resultados de un único censo no representan las abundancias de las gaviotas que invernan en la costa vasca, por lo que se recomienda llevar a cabo varios censos con el fin de estimar un valor medio junto a su incertidumbre asociada. Sería interesante, además, ampliar el periodo de censo a otros días de la semana con el fin de conocer posibles efectos de factores como la actividad pesquera en la abundancia.

Desde un punto de vista biogeográfico amplio, la abundancia de gaviotas en la costa vasca es poco relevante si la comparamos con las cifras para el conjunto de toda la costa del norte de España. Así, según el censo de 2009 (Molina, 2009), el sector atlántico (desde Euskadi hasta Galicia) acogió casi 120000 patiamarillas, unas 70000 sombrías y casi 35000 reidoras. Es cierto que este censo se llevó a cabo, también, en zonas de interior, si bien la mayor parte de las localidades que se reportan se sitúan a lo largo de la costa. Aunque, obviamente, la abundancia de gaviotas puede variar entre años, estos datos sí permiten contextualizar la magnitud de la abundancia entre zonas. En este contexto, la costa vasca no albergaría si quiera el $5 \%$ de la abundancia de gaviotas de toda la costa norte de España. A escala de ruta migratoria (ruta del Atlántico del Paleártico Occidental), ninguna de las especies más abundantes (gaviota sombría, reidora y patiamarilla) alcanzan si quiera el $1 \%$ de la población de la zona biogeográfica (Wetlands International, 2012).

Si acotamos el censo de 2009 al área cantábrica desde Euskadi hasta Asturias, con una estima de unas 50000 gaviotas entre reidoras, sombrías y patiamarillas (Molina, 2009), la costa vasca, todavía, no llegaría al 10\% del total. Esta importancia, baja en 
el contexto cantábrico, se debe probablemente a varias causas, entre ellas: (1) el grueso de la población de patiamarillas, que además son sedentarias (Munilla, 1997), se sitúa en Galicia; (2) Galicia, además, cuenta con una costa con una alta productividad, por lo que es una zona más atractiva para las aves marinas, láridos incluidos; (3) Euskadi, asimismo, tiene la línea de costa más corta de todas las regiones del área cantábrica (Euskadi, 176 km; Cantabria, casi 600 km; Galicia, casi 1500 km), por lo que tampoco es extraño que, en concordancia, el número de gaviotas que acoge sea, también, más bajo; (4) no hay que descartar que una fracción de las gaviotas que frecuentan la costa vasca realice, también, desplazamientos al interior (e.g. para alimentarse en vertederos, prados o ríos; Egunez et al., 2017, Arizaga et al., 2018).

En cuanto a las zonas más importantes para la invernada de gaviotas en la costa vasca, destacan Donostia, Txingudi, Urdaibai y Ondarroa. Cabe destacar que las dos colonias más grandes de gaviota patiamarilla en la costa vasca se sitúan en Donostia (colonias de Ulia y Santa Clara) y Urdaibai (Izaro) (Arizaga et al., 2009) por lo que, dado el carácter sedentario de la población (Arizaga et al., 2010, Arizaga et al., 2014), no es de extrañar que las abundancias más elevadas se sitúen cerca de tales colonias. Sorprende, no obstante, el hecho de que la abundancia de gaviotas en Getaria fuera muy baja (Fig. 4), aunque en Getaria hay una colonia de gaviota patiamarilla estimada en más de 200 parejas (Zorrozua et al., 2020). Las gaviotas de ésta, además, dependen en buena parte de los recursos que obtienen, aparentemente, en el propio puerto de Getaria (Arizaga et al., 2013, Zorrozua et al., 2020). El hecho de que el censo se hiciera en sábado, cuando no existe actividad comercial en el puerto, pudo influir decisivamente en el resultado, ya que las gaviotas alteran sus hábitos de búsqueda de alimento según sean fines de semana o días laborables (e.g., Bécares et al., 2015). El motivo de hacer el trabajo en sábado vino dado por una cuestión de disponibilidad de personal para censar. En el futuro, será necesario determinar hasta qué punto esta decisión podría sesgar los resultados (tal vez la abundancia de gaviotas podría aumentar en días laborables).

El 66,5\% de la abundancia la acumula la gaviota patiamarilla; sobre el total estimado, esto supone ca. 3000 ejemplares (para más detalles ver, también, la Fig. 2). Este resultado contrasta con los resultados de la década de 1980, en los que se obtuvo para la costa vasca unas 2900 gaviotas reidoras, algo más de 3000 patiamarillas (entonces clasificadas como argénteas) y apenas unas 50 sombrías (Bermejo et al., 1986). En consecuencia, cabe destacar el descenso notable de gaviota reidora, cuyo tamaño poblacional actual, estimado en 950 (IC95\%: 500-1700) ejemplares es, centrándonos en el límite superior del intervalo de confianza al 95\%, un 60\% del que se obtuvo en la década de 1980. Las causas que explican tal declive son desconocidas, entre ellas podría subyacer, entre otras, un cambio en el área de distribución no reproductora de las poblaciones que invernaban en la región. Otras causas, como cambios del hábitat en la región o declives poblacionales, serían aparentemente menos probables. Desde la década de 1980, la calidad del hábitat que ocupan las gaviotas reidoras en la costa 
vasca (estuarios) ha mejorado debido, entre otros, a los esfuerzos que se han desarroIlado en materia de depuración de aguas (Borja et al., 2004, Borja et al., 2010). Por otro lado, a escala europea la población de gaviota reidora no está en declive, aunque sí es fluctuante (Staneva and Burfield, 2017).

En la actualidad, el tamaño de las colonias que se distribuyen a lo largo de la costa vasca asciende a 2300 parejas de adultos reproductores, esto es, 4600 ejemplares. A ello hay que añadir la población subadulta no reproductora. Si asumimos un promedio de 2 huevos eclosionados/nido (S. Delgado, no publ.), un valor medio de supervivencia de 0,4 durante el periodo de permanencia de pollos en el nido (colonia) (Delgado and Arizaga, 2017) y supervivencia media anual de 0,5 para ejemplares en su primer año y 0,8 para los años siguientes (Juez et al., 2015), obtendríamos, grosso modo, unos 3500 ejemplares más que, en total, suman unas 8100 gaviotas patiamarillas de origen 'local' (cohortes: comienzo del primer año, $2300 \times 2 \times 0,4=1840$ ejemplares; comienzo del $2^{\circ}$ año, $1840 \times 0,5=920$ ejemplares; comienzo del 3er año, $920 \times 0,8=760$ ejemplares; edad adulta: 4600 ejemplares). Es cierto, no obstante, que el valor real puede situarse muy por debajo, ya que la productividad en varias colonias es posiblemente inferior (e.g., en Izaro) (Galarza, 2015), así como los valores de supervivencia (Juez et al., 2015). Aun así parece que la población local excedería el número de gaviotas patiamarillas estimado en toda la costa vasca en invierno. Esta diferencia podría ser debida a varias causas, entre otras: (1) una fracción del contingente 'local' de patiamarillas pasa el invierno en otras zonas, sobre todo aves subadultas (Arizaga et al., 2010, Arizaga et al., 2015); (2) una parte del área de estudio no se censó, por lo que no debe descartarse la omisión de cierto número de ejemplares. El valor medio anual para estas zonas sin censar (estuarios de Ea, Bakio, Plentzia y Barbadun) durante el periodo 1992-2013 suma, no obstante, 155 patiamarillas (ver los anexos en Barainka and Arizaga, 2015), por lo que no parece que estas zonas sin censar fueran relevantes en el contexto de la costa vasca; (3) las patiamarillas que se ven en la costa explotan otros hábitats, como vertederos y el mar (Jordi et al., 2014, Arizaga et al., 2017, Egunez et al., 2017) que quedan al margen de la zona muestreada en este estudio. Por otro lado, además hay que considerar que la costa vasca recibe en invierno gaviotas patiamarillas de origen mediterráneo (Galarza et al., 2012), que por tanto se suman al contingente de aves 'locales'. El número de ejemplares de este origen se desconoce, si bien estudios llevados a cabo en vertederos de Gipuzkoa y su entorno indican que debe ser marginal en relación al contingente de aves 'locales' (Jordi et al., 2014). En todo caso, el número de gaviotas patiamarillas presentes en la costa vasca en un instante dado es inferior al stock de patiamarillas que habría en la región (incluidos el interior de las dos provincias estudiadas). Tentativamente, este stock podría como mínimo duplicar el número de gaviotas patiamarillas observadas en la costa en un momento dado.

Estructuralmente, hay dos tipos de zonas (estuarios) en la costa vasca: (1) aquellas en las que la gaviota reidora muestra niveles de abundancia relativa comparables a los 
de la gaviota patiamarilla y (2) aquellas zonas donde domina esta última (que por otro lado son la mayoría). Esta estructura ya se había descrito antes (Barainka and Arizaga, 2015), si bien en este artículo se analizaban, además, el resto de especies acuáticas. Desde el punto de vista de la conservación de la biodiversidad, los estuarios que albergan más gaviotas reidoras son, también, los más ricos en especies. Algunos de éstos están, además, más naturalizados que otros donde domina la gaviota patiamarilla (e.g., Txingudi o el estuario del río Oria), aunque este no es el caso de todos ellos (e.g., Mutriku es un estuario intensamente urbanizado). Las gaviotas son especies con una gran capacidad para explotar recursos de origen humano, por lo que son habituales en puertos y lugares antropizados de la costa, donde encuentran gran cantidad de alimento (Belant et al., 1998, Duhem et al., 2003, Moreno et al., 2009, Arizaga et al., 2013, Egunez et al., 2017, Zorrozua et al., 2020). Así, el grado de naturalización de un estuario no parece, en principio, un factor influyente en la estructuración del ensamblado de este tipo de aves en la costa vasca (Fig. 5).

Al margen de tres especies (gaviotas reidora, sombría y patiamarilla), el resto de gaviotas son muy raras desde un punto de vista cuantitativo (Fig.6). En términos globales, apenas acumulan en torno al $5 \%$ de la abundancia como máximo, si bien lo habitual es que no lleguen si quiera al $1 \%$. En este contexto, la presencia de estas especies sería irrelevante desde un punto de vista ecosistémico y biogeográfico. En general, se trata de ejemplares de especies muy nórdicas que de vez en cuando sobrepasan el límite septentrional de su área de distribución en periodo no reproductor (Olsen and Larson, 2004), de especies muy pelágicas que sólo se avistan en la costa en caso de temporales o condiciones meteorológicas muy particulares, o de especies muy orientales que rara vez alcanzan el Cantábrico occidental. Dentro de este último caso se situaría la gaviota cáspica L. cachinnans Pallas 1811, que parece que, aun siendo escasa, alcanza máximos de abundancia en el Cantábrico oriental, respecto al Cantábrico occidental (G. Martín, com. pers.).

En conclusión, la abundancia global de gaviotas invernantes en la costa vasca se estima en unos 4500 ejemplares, de los que algo más del 65\% son gaviotas patiamarillas. Existe, asociado a esta estima, un rango de error amplio, debido a la alta variabilidad en el número de ejemplares, tanto entre zonas como entre días. El valor de un censo único en enero, o en cualquier otro momento del invierno es, en consecuencia, poco representativo. En un contexto cantábrico, además, la costa vasca es poco relevante como zona de invernada para gaviotas, si bien juega un papel clave para la población local residente de patiamarillas (Arizaga et al., 2010, Egunez et al., 2017) y, quizás, algunas especies escasas en el ámbito cantábrico, pero que pueden tener una mayor representatividad en el Cantábrico oriental, como tal vez la gaviota cáspica. A escalas mayores, incluida la de la ruta del Atlántico del Paleártico Occidental (Wetlands International, 2012), la importancia de la costa vasca para las gaviotas es marginal. 


\section{Agradecimientos}

Este proyecto se desarrolló por voluntarios que asumieron por sí mismos los costes de los desplazamientos que se hubieron de hacer para llevar a cabo los censos. L. M. Carrascal hizo una revisión muy valiosa de una primera versión del trabajo, que contribuyó a mejorar los modelos estadísticos. A. Bermejo, C. Carboneras y B. Martín revisaron la versión del trabajo enviada a publicar.

\section{Bibliografía}

Akaike, H., 2011. Akaike's Information Criterion. In: Lovric, M. (Eds.), International Encyclopedia of Statistical Science, 25-25. Springer Berlin Heidelberg, Berlin.

Arizaga, J., Aldalur, A., Herrero, A., Cuadrado, J., Díez, E., Crespo, A., 2014. Foraging distances of a resident yellow-legged gull (Larus michahellis) population in relation to refuse management on a local scale. Eur. J. Wildlife Res. 60, 171-175.

Arizaga, J., Galarza, A., Herrero, A., Hidalgo, J., Aldalur, A., 2009. Distribución y tamaño de la población de la Gaviota Patiamarilla Larus michahellis lusitanius en el País Vasco: tres décadas de estudio. Rev. Cat. Ornitol. 25, 32-42.

Arizaga, J., Herrero, A., Aldalur, A., Cuadrado, J.F., 2015. Primeras observaciones de gaviotas patiamarillas Larus michahellis Naumann, 1840 de origen cantábrico en la cuenca del río Ebro. Munibe, Cienc. nat. 63, 155-162.

Arizaga, J., Herrero, A., Galarza, A., Hidalgo, J., Aldalur, A., Cuadrado, J.F., Ocio, G., 2010. First-year movements of Yellow-legged Gull (Larus michahellis lusitanius) from the southeastern Bay of Biscay. Waterbirds 33, 444-450.

Arizaga, J., Jover, L., Aldalur, A., Cuadrado, J.F., Herrero, A., Sanpera, C., 2013. Trophic ecology of a resident Yellow-legged Gull (Larus michahellis) population in the Bay of Biscay. Mar. Environ. Res. 87-88, 19-25.

Arizaga, J., Laso, M., Zorrozua, N., Delgado, S., Aldalur, A., Herrero, A., 2017. Uso del espacio por adultos de gaviota patiamarilla Larus michahellis Naumann, 1840 durante el periodo reproductor: resultados preliminares en relación al uso de vertederos. Munibe, Cienc. nat. 65, 67-80.

Arizaga, J., Zorrozua, N., Egunez, A. 2018. Between the land and sea: how yellow-legged gulls have changed their dependence on marine food in relation to landfill management. In: Mikkola, H. (Eds.), Seabirds, 67-78. InTech Open.

Barainka, P., Arizaga, J., 2015. Distribution and population trends of waterbird species wintering in Basque estuaries (North of Spain): a 22-year study. Munibe, Cienc. nat. 63, 2947.

Bécares, J., García-Tarrasón, M., Villero, D., Bateman, S., Jover, L., García-Matarranz, V., Sanpera, C., Arcos, J. M. 2015. Modelling terrestrial and marine foraging habitats in breeding Audouin's Gulls Larus audouinii: timing matters. Plos One 10, e0120799. 
Belant, J.L., Ickes, S.K., Seamans, T. W. 1998. Importance of landfills to urban-nesting herring and ring-billed gulls. Landscape Urban Plan. 43, 11-19.

Bermejo, A., Carrera, E., De Juana, E., Teixeira, A.M., 1986. Primer censo general de gaviotas y charranes (Laridae) invernantes en la península ibérica. Ardeola 33, 47-68.

Bermejo, A., Carrera, E., De Juana, E., Teixura, A.M., 1984. Primer censo de láridos invernantes en la Península Ibérica, Islas Canarias y Ceuta. La Garcilla 61, 30-33.

Borja, Á., Dauer, D., Elliott, M., Simenstad, C., 2010. Medium- and long-term recovery of estuarine and coastal ecosystems: patterns, rates and restoration effectiveness. Estuar. Coast. 33, 1249-1260.

Borja, Á., Franco, J., Valencia, V., Bald, J., Muxika, I., Belzunce, M.J., Solaun, O., 2004. Implementation of the European water framework directive from the Basque country (northern Spain): a methodological approach. Marine Pol. Bull. 48, 209-218.

Burnham, K.P., Anderson, D.R. 1998. Model Selection and Inference. A Practical Information Theoretic Approach. Springer-Verlag, New York.

Delgado, S., Arizaga, J., 2017. Pre-fledging survival in a Yellow-legged Gull Larus michahellis population in northern Iberia is mostly determined by hatching date. Bird Stud. 64, 132-137.

Duhem, C., Vidal, E., Legrand, J., Tatoni, T., 2003. Opportunistic feeding responses of the Yellow-legged Gull Larus michahellis to accessibility of refuse dumps. Bird Stud. 50, 6167.

Egunez, A., Zorrozua, N., Aldalur, A., Herrero, A., Arizaga, J., 2017. Local use of landfills by a yellow-legged gull population suggests distance-dependent resource exploitation. J. Avian Biol. 49, e01455.

Elkins, N., 1983. Weather and bird behaviour. Poyser, London.

Fernández, J.M., Gracianteparaluceta, A., Planillo, A., 2012. Abundancia, distribución y tendencia de las poblaciones de aves acuáticas invernantes en la Comunidad Autónoma del País Vasco. Departamento de Medio Ambiente y Política Territorial (Gobierno Vasco), Inédito.

Galarza, A., 2015. ¿Está disminuyendo la población de gaviota patiamarilla cantábrica Larus michahellis lusitanius Naumann, 1840? Censo 2013/2014 de Bizkaia (País Vasco)? Munibe, Cienc, nat. 63, 135-143.

Galarza, A., Herrero, A., Domínguez, J.M., Aldalur, A., Arizaga, J., 2012. Movements of Mediterranean Yellow-legged Gulls Larus michahellis to the Bay of Biscay. Ring. Migr. 27, 26-31.

Hammer, Ø., Harper, D.A.T., Ryan, P.D., 2001. PAST: Palaeontological Statistics software package for education and data analysis. Palaentologia Electronica 4.

Jordi, O., Herrero, A., Aldalur, A., Cuadrado, J. F., Arizaga, J. 2014. The impact of nonlocal birds on yellow-legged gulls (Larus michahellis) in the Bay of Biscay: a dump-based assessment. Anim. Biodiv. Conserv. 37, 183-190. 
Juez, L., Aldalur, A., Herrero, A., Galarza, A., Arizaga, J. 2015. Effect of age, colony of origin and year on survival of Yellow-Legged Gulls Larus michahellis in the Bay of Biscay. Ardeola 62, 139-150.

Magurran, A. E., McGill, B.J., 2011. Biological Diversity - Frontiers in measurement and assessment. Oxford University Press, Oxford.

Molina, B.E., 2009. Gaviota reidora, sombría y patiamarilla en España. Población en 20072009 y método de censo. SEO/BirdLife, Madrid.

Moreno, R., Jover, L., Munilla, I., Velando, A., Sanpera, C., 2009. A three-isotope approach to disentangling the diet of a generalist consumer: the yellow-legged gull in northwest Spain. Marine Biol. 157, 545-553.

Munilla, I., 1997. Desplazamientos de la Gaviota Patiamarilla (Larus cachinnans) en poblaciones del norte de la Península Ibérica. Ardeola 44, 19-26.

Olsen, K.M., Larson, H., 2004. Gulls of Europe, Asia and North America. Christopher Helm, London.

R Core Team, 2014. R: A language and environment for statistical computing. ISBN 3900051-07-0, Vienna, Austria.

Rouco, M., Copete, J.L., De Juana, E., Gil-Velasco, M., Lorenzo, J.A., Martín, M., Milá, B., Molina, B., Santos, D.M., 2019. Lista de las aves de España. Edición de 2019. SEO/BirdLife, Madrid.

SEO/BirdLife. 2012. Atlas de las aves en invierno en España 2007-2010. Ministerio de Agricultura, Alimentación y Medio Ambiente-SEO/BirdLife, Madrid.

Staneva, A., Burfield, I., 2017. European birds of conservation concern: populations, trends and national responsibilities. BirdLife International.

Wetlands International, 2012. Waterbird Population Estimates, 5th Ed. Wetlands International, Wageningen, The Netherlands.

Zorrozua, N., Egunez, A., Aldalur, A., Galarza, A., Díaz, B., Hidalgo, J., Jover, L., Sanpera, C., Castège, I., Arizaga, J., 2020. Evaluating the effect of distance to different food subsidies on the trophic ecology of an opportunistic seabird species. J. Zool. 311, 45-55.

\section{常}

Fecha de recepción/ Date of reception: 27/04/2020

Fecha de aceptación / Date of acceptance: 09/07/2020

Editor Asociado / Associate editor: Beatriz Martín 


\section{Anexos}

\begin{tabular}{|c|c|c|}
\hline Código & Unidad & Sectores \\
\hline Txin & Txingudi & $\begin{array}{l}\text { Hondarribia-Playa / Hondarribia-Po Butrón / Hondarribia-Puerto / Hon- } \\
\text { darribia-Puerto deportivo / Beltzenia / Irun-Plaiaundi /Irun-Osinbiribil. }\end{array}$ \\
\hline Pasa & Pasaia & Punto de censo único (puerto). \\
\hline Dono & Donostia & $\begin{array}{l}\text { Desembocadura Urumea / Urumea-Frente a Hotel María Cristina / } \\
\text { Urumea-Sector Puente } 2 \text { a } 3 \text { / Urumea-Sector Puente } 3 \text { a } 4 \text { / } \\
\text { Urumea-Sector Puente } 4 \text { a } 5 \text { / Urumea-Sector Puente } 5 \text { a } 6 \text { / } \\
\text { Urumea-Sector Puente Hierro a Puente de acceso a Cristina-Enea / } \\
\text { Urumea-Puente Cristina Enea a Puente Egia / Urumea-Puente Egia } \\
\text { a Puente Tren Loiola / Urumea-Barrio de Loiola a Txomin / Urumea- } \\
\text { Playazos de Martutene. }\end{array}$ \\
\hline Oria & Oria & $\begin{array}{l}\text { Oria-Sector Puente Viaducto a desembocadura (incl. playas) / Oria- } \\
\text { Sector Puente Viaducto a Puente Orio / Oria-Sector Puente Orio a } \\
\text { Motondo / Oria (medio) Aguinaga / Oria (medio) Lasarte-Oria. }\end{array}$ \\
\hline Zara & Zarautz & Iñurritza / Tramos costeros. \\
\hline Geta & Getaria & Playas / Puerto. \\
\hline Zuma & Zumaia & Marismas de Santiago / Bedua. \\
\hline Mutr & Mutriku & Puerto / Tramos costeros (Hondarbeltz). \\
\hline Onda & Ondarroa & Puerto / Pueblo y estuario / Posadero de gaviotas. \\
\hline Leke & Lekeitio & Karraspio / Mari Errota. \\
\hline Urda & Urdaibai & Canal principal / Izaro / Laida / Laga. \\
\hline Berm & Bermeo & Punto de censo único (puerto). \\
\hline Abra & Bilbao-Abra & $\begin{array}{l}\text { Muelle de cruceros / La Bola / Puerto deportivo (Getxo) / Santurtzi / } \\
\text { Puerto de Zierbana. }\end{array}$ \\
\hline
\end{tabular}

Anexo 1.- Unidades de muestreo y sectores de censo de gaviotas. Para conocer en detalle la localización de cada sector de censo véase la página de Ornitho Euskadi (www.ornitho.eus).

Annex 1.- Gull sampling localities (some localities were divided into sectors). For more detailed data on each sampling location, see the portal Ornitho Euskadi. (www.ornitho.eus). 


\begin{tabular}{|c|c|c|c|c|c|c|c|c|c|c|c|c|c|c|}
\hline Especies & TXIN & PASA & DONO & ORIA & ZARA & GETA & ZUMA & DEBA & MUTR & ONDA & LEKE & URDA & BERM & 1) ABRA \\
\hline R. tridactyla & & & & & & \begin{tabular}{|c|}
2 \\
$0.3 \%$ \\
$(0-1)$
\end{tabular} & & & & & & & & \\
\hline X. sabini & & & & & & & & & & & & & & $\begin{array}{c}1 \\
0.1 \% \\
(0-1)\end{array}$ \\
\hline C. ridibundus & \begin{tabular}{|c|}
2323 \\
$49.6 \%$ \\
$(179-499)$ \\
\end{tabular} & $\begin{array}{c}354 \\
26.2 \% \\
(8-100)\end{array}$ & \begin{tabular}{|l|}
1051 \\
$22.6 \%$ \\
$(32-217)$
\end{tabular} & $\begin{array}{l}935 \\
51.0 \% \\
(70-220)\end{array}$ & $\begin{array}{c}1 \\
0.1 \% \\
(0-1)\end{array}$ & $\begin{array}{c}8 \\
1.1 \% \\
(0-3)\end{array}$ & $\begin{array}{c}379 \\
15.5 \% \\
(14-80)\end{array}$ & \begin{tabular}{|c|}
107 \\
$19.0 \%$ \\
$(0-28)$
\end{tabular} & \begin{tabular}{c|}
450 \\
$56.0 \%$ \\
$(0-350)$ \\
\end{tabular} & $\begin{array}{c}683 \\
27.2 \% \\
(26-149)\end{array}$ & $\begin{array}{c}1 \\
0.1 \% \\
(0-1)\end{array}$ & \begin{tabular}{|c|}
97 \\
$3.8 \%$ \\
$(9-65)$
\end{tabular} & \begin{tabular}{|c|}
8 \\
$1.5 \%$ \\
$(0-8)$ \\
\end{tabular} & $\begin{array}{c}485 \\
26.1 \% \\
(21-121)\end{array}$ \\
\hline H. minutus & & & & & & & & & & & & & & $\begin{array}{c}7 \\
0.4 \% \\
(0-7)\end{array}$ \\
\hline L. melanocephalus & \begin{tabular}{|c|}
103 \\
$2.2 \%$ \\
$(0-56)$ \\
\end{tabular} & & $\begin{array}{c}4 \\
0.1 \% \\
(0-1)\end{array}$ & $\begin{array}{c}2 \\
0.1 \% \\
(0-1)\end{array}$ & $\begin{array}{c}4 \\
0.4 \% \\
(0-4)\end{array}$ & $\begin{array}{c}1 \\
0.1 \% \\
(0-1)\end{array}$ & $\begin{array}{c}2 \\
0.1 \% \\
(0-1)\end{array}$ & \begin{tabular}{|l|}
23 \\
$4.1 \%$ \\
$(0-21)$ \\
\end{tabular} & \begin{tabular}{|c|}
6 \\
$0.7 \%$ \\
$(0-6)$ \\
\end{tabular} & & $\begin{array}{c}3 \\
0.2 \% \\
(0-2)\end{array}$ & & & \begin{tabular}{|l|}
210 \\
$11.3 \%$ \\
$(0-170)$
\end{tabular} \\
\hline L. canus & & & & $\begin{array}{c}1 \\
<0.1 \% \\
(0-1)\end{array}$ & & & & & & $\begin{array}{c}1 \\
<0.1 \% \\
(0-1)\end{array}$ & & & & $\begin{array}{c}4 \\
<0.1 \% \\
(0-4)\end{array}$ \\
\hline L. delawarensis & & & & & & & & & & & & & & $\begin{array}{c}4 \\
0.2 \% \\
(0-1)\end{array}$ \\
\hline L. marinus & $\begin{array}{c}55 \\
1.2 \% \\
(3-12)\end{array}$ & $\begin{array}{c}4 \\
0.3 \% \\
(0-2)\end{array}$ & $\begin{array}{c}1 \\
<0.1 \% \\
(0-1)\end{array}$ & $\begin{array}{l}7 \\
0.4 \% \\
(0-3)\end{array}$ & $\begin{array}{c}1 \\
0.1 \% \\
(0-1)\end{array}$ & $\begin{array}{c}2 \\
0.3 \% \\
(0-1)\end{array}$ & $\begin{array}{c}4 \\
0.2 \% \\
(0-2)\end{array}$ & & \begin{tabular}{c|}
1 \\
$0.1 \%$ \\
$(0-1)$
\end{tabular} & $\begin{array}{c}7 \\
0.3 \% \\
(0-3)\end{array}$ & $\begin{array}{c}2 \\
0.1 \% \\
(0-1)\end{array}$ & $\begin{array}{c}3 \\
0.1 \% \\
(0-1)\end{array}$ & & $\begin{array}{c}32 \\
1.7 \% \\
(0-22)\end{array}$ \\
\hline L. glaucoides & & & & & & & & & & $\begin{array}{c}1 \\
<0.1 \% \\
(0-1) \\
\end{array}$ & & & & \\
\hline L. argentatus & $\begin{array}{c}6 \\
0.1 \% \\
(0-2)\end{array}$ & $\begin{array}{c}5 \\
0.4 \% \\
(0-3)\end{array}$ & \begin{tabular}{|c|}
2 \\
$<0.1 \%$ \\
$(0-1)$
\end{tabular} & \begin{tabular}{|c|}
4 \\
$0.2 \%$ \\
$(0-2)$
\end{tabular} & & $\begin{array}{c}2 \\
0.3 \% \\
(0-1)\end{array}$ & $\begin{array}{c}2 \\
0.1 \% \\
(0-1)\end{array}$ & & $\begin{array}{c}1 \\
0.1 \% \\
(0-1)\end{array}$ & $\begin{array}{c}9 \\
0.4 \% \\
(0-3)\end{array}$ & & & & $\begin{array}{c}9 \\
0.5 \% \\
(0-9)\end{array}$ \\
\hline L. cachinnans & $\begin{array}{c}1 \\
<0.1 \% \\
(0-1)\end{array}$ & & $\begin{array}{c}1 \\
<0.1 \% \\
(0-1)\end{array}$ & & & $\begin{array}{c}2 \\
0.3 \% \\
(0-1)\end{array}$ & & $\begin{array}{c}1 \\
0.2 \% \\
(0-1)\end{array}$ & & $\begin{array}{c}2 \\
0.1 \% \\
(0-1)\end{array}$ & & & & \\
\hline L. michahellis & $\begin{array}{c}2001 \\
42.7 \% \\
(57-930)\end{array}$ & $\begin{array}{c}936 \\
69.3 \% \\
(85-200)\end{array}$ & \begin{tabular}{|l|}
3527 \\
$75.9 \%$ \\
$(323-663)$
\end{tabular} & $\begin{array}{c}859 \\
46.9 \% \\
(71-204\end{array}$ & $\begin{array}{c}803 \\
81.0 \% \\
(0-400)\end{array}$ & $\begin{array}{c}613 \\
85.5 \% \\
(29-165)\end{array}$ & $\begin{array}{c}1950 \\
79.9 \% \\
(75-701)\end{array}$ & \begin{tabular}{|c|}
332 \\
$58.9 \%$ \\
$(2-280)$
\end{tabular} & \begin{tabular}{|c|}
340 \\
$42.3 \%$ \\
$(9-126)$
\end{tabular} & $\begin{array}{l}1710 \\
68.0 \% \\
(46-457)\end{array}$ & $\begin{array}{c}1015 \\
75.7 \% \\
(60-300)\end{array}$ & $\begin{array}{c}2115 \\
82.5 \% \\
(251-1004)\end{array}$ & $\begin{array}{c}525 \\
95.8 \% \\
70-286\end{array}$ & $\begin{array}{l}1011 \\
54.4 \% \\
(16-582)\end{array}$ \\
\hline L. fuscus & \begin{tabular}{|c|}
195 \\
$4.2 \%$ \\
$(4-72)$ \\
\end{tabular} & $\begin{array}{c}52 \\
3.8 \% \\
(1-25)\end{array}$ & \begin{tabular}{|c|}
59 \\
$1.3 \%$ \\
$(2-15)$
\end{tabular} & $\begin{array}{c}25 \\
1.4 \% \\
(0-17)\end{array}$ & $\begin{array}{c}182 \\
18.4 \% \\
(0-161)\end{array}$ & $\begin{array}{c}87 \\
12.1 \% \\
(0-75)\end{array}$ & $\begin{array}{c}104 \\
4.3 \% \\
(1-55)\end{array}$ & \begin{tabular}{|c|}
101 \\
$17.9 \%$ \\
$(0-95)$
\end{tabular} & $\begin{array}{c}5 \\
0.6 \% \\
(0-3)\end{array}$ & $\begin{array}{c}103 \\
4.1 \% \\
(2-33)\end{array}$ & $\begin{array}{c}249 \\
18.6 \% \\
(0-200)\end{array}$ & \begin{tabular}{|c|}
351 \\
$13.7 \%$ \\
$(1-234)$
\end{tabular} & $\begin{array}{c}15 \\
2.7 \% \\
(0-11)\end{array}$ & $\begin{array}{c}94 \\
5.1 \% \\
(2-46)\end{array}$ \\
\hline $\begin{array}{l}\text { L. michahellis/ } \\
\text { fuscus }\end{array}$ & & & & & & & & & & & $\begin{array}{c}70 \\
5.2 \% \\
(0-70)\end{array}$ & & & \\
\hline
\end{tabular}

Anexo 2.- Especies de gaviotas detectadas durante el periodo de censo en la costa vasca, en el invierno 2019/20. Se muestran, para cada zona, el número global de aves censadas, el porcentaje de la abundancia, respecto a este número global de cada una de las especies y el número mínimo y máximo de individuos contados en un censo único (en paréntesis). Especies según orden taxonómico (Rouco et al., 2019).

Annex 2.- Gull species detected on the Basque coast during the winter 2019/20. For each zone we show the overall number of bird counts, their abundance in percentage, and the minimum and maximum number of individual gulls detected in a single census (in parenthesis). Species ordered taxonomically (Rouco et al., 2019). 